\title{
Differential Regional Dysfunction of the Hippocampal Formation among Elderly with Memory Decline and Alzheimer's Disease
}

\author{
Scott A. Small, MD, ${ }^{*} \dagger \ddagger$ Gerard M. Perera, MS, $\$$ Robert DeLaPaz, MD, $\$$ Richard Mayeux, MD, MSc, ${ }^{*} \dagger \ddagger \|_{\mathfrak{g}}$ \\ and Yaakov Stern, $\mathrm{PhD}^{*}+\ddagger$ g
}

\begin{abstract}
The hippocampal formation is composed of separate anatomical regions interconnected to form a circuit, and investigating abnormal hippocampal function is most revealing at the level of these regions. Until recently, regional analysis of the hippocampal formation could be performed only in animals or in human postmortem tissue. Here, we report a method using functional magnetic resonance imaging that evaluates the hippocampal regions in vivo, and we use this method to study elderly with normal memory, with isolated memory decline, and with probable Alzheimer's disease (AD). Although age-related memory decline occurs commonly, the cause of this decline remains unknown, with disagreement as to whether this decline represents one or more etiologies. Analysis revealed two distinct patterns of regional dysfunction among elderly with isolated memory decline-one pattern similar to that found in elders with $\mathrm{AD}$, involving all hippocampal regions, and a second pattern with dysfunction restricted to only one hippocampal region, the subiculum. These results offer direct evidence of hippocampal dysfunction associated with memory decline in the elderly, and implicate both predementia $\mathrm{AD}$ and non- $\mathrm{AD}$ processes as possible underlying causes.
\end{abstract}

Small SA, Perera GM, DeLaPaz R, Mayeux R, Stern Y. Differential regional dysfunction of the hippocampal formation among elderly with memory decline and Alzheimer's disease. Ann Neurol 1999;45:466-472

Amnestic patients with discrete lesions, ${ }^{1,2}$ lesion studies in animals, ${ }^{3}$ and recent functional neuroimaging studies $^{4,5}$ have established a role for the hippocampal formation in consolidating new declarative memories. Increased interest in the hippocampus has led to a more detailed understanding of its microanatomy and physiology. In its transverse axis, the hippocampal formation is made up of different regions that are interconnected to form a complex circuit. ${ }^{6}$ These regions include the entorhinal cortex that serves as the main gateway into the hippocampal circuit, the "hippocampus proper" composed of the dentate gyrus and cornus ammonis (CA) subfields, and the subiculum. The physiological properties of hippocampal neurons have also been investigated, and these efforts have revealed mechanisms of cellular plasticity, including long-term potentiation. ${ }^{7}$ Synapses in different hippocampal regions use varying forms of long-term potentiation, ${ }^{8}$ and some of these may underlie the mnemonic function of the hippocampus.

Thus, the hippocampal formation is not a simple unitary structure. Rather, it is anatomically complex and physiologically diverse. When investigating mechanisms of memory dysfunction, evaluating the hippocampus globally may be incompatible with its level of complexity. The advantages of a regional approach to hippocampal function have been exploited in animal studies and, in a more limited fashion, in human postmortem studies. ${ }^{10-12}$ Until recently, however, in vivo methods did not possess adequate spatial resolution to perform regional analysis of the hippocampal formation. Functional magnetic resonance imaging (fMRI), a relatively new neuroimaging technique, ${ }^{13}$ has enhanced spatial resolution that is within the dimensions of the hippocampal subregions. Recent structural $^{14}$ and functional MRI ${ }^{15}$ studies have successfully demonstrated its ability to selectively evaluate the different regions. In the current study we use an fMRI protocol that was specifically developed for hippocampal regional analysis to investigate age-related memory decline.

Memory decline with age is common, with some re-
From the ${ }^{*}$ Gertrude H. Sergievsky Center, Departments of $†$ Neurology, "Psychiatry, and $₫$ Radiology, $¥$ Taub Center for Alzheimer’s Disease Research in the City of New York, and IDivision of Epidemiology, School of Public Health, Columbia University College of Physicians and Surgeons, New York, NY.
Received Aug 18, 1998, and in revised form Nov 25. Accepted for publication Dec 4, 1998.

Address correspondence to Dr Small, Gertrude H. Sergievsky Center, 630 West 168 Street, New York, NY 10032. 
ports suggesting a prevalence of $>40 \%$ among individuals of $>60$ years. ${ }^{16}$ One component of this decline affects declarative memory function ${ }^{17,18}$ and it is thought to localize to the hippocampal formation. ${ }^{19}$ Because of a bimodal distribution of memory scores in aging animals and humans, ${ }^{20}$ where many older individuals perform as well as younger controls, memory decline may not be an inevitable consequence of aging. What, then, are the possible causes of age-related memory decline? The first brain structure to be targeted by Alzheimer's disease (AD) is the hippocampal formation, ${ }^{21}$ and correspondingly, prospective studies have found that memory deficits are the first signs of $\mathrm{AD} .{ }^{22}$ Recent studies have shown that entorhinal cell loss occurs in the predementia stage of the disease. ${ }^{10}$ It is likely, therefore, that early $\mathrm{AD}$ is one contributing cause of age-related memory decline. Although the association between age-related memory decline and $\mathrm{AD}$ awaits further confirmation, a more contentious issue is whether $\mathrm{AD}$ can explain all cases of elderly patients with memory decline. This is particularly important, as there are age-dependent changes in some physiological processes, such as hormone levels and cerebrovascular supply, that can affect the hippocampal formation and result in memory impairment. ${ }^{23-26}$ Although these processes may interact with $\mathrm{AD}$, as modifiers or even triggers of disease, recent postmortem studies have documented age-related cell loss in the hippocampal formation among brains free of $\mathrm{AD}$ pathology. ${ }^{11,12} \mathrm{~A}$ limitation of these studies is that antemortem evaluation of memory function were not performed. Aside from structural lesions, non-AD processes might also cause functional damage to hippocampal neurons. Extensive animal research has documented age-dependent changes in the physiological properties of hippocampal neurons, ${ }^{9,19}$ and some may explain non-AD memory decline found pervasively in aging animals.

Although both early $\mathrm{AD}$ and non-AD processes involve the hippocampal formation, they appear to target different hippocampal subregions. The first hippocampal region to be targeted by $\mathrm{AD}$ is the entorhinal cortex, as evidenced by changes in synaptic integrity, ${ }^{26}$ reduction in cell density, ${ }^{10}$ and the formation of neurofibrillary tangles. ${ }^{21}$ In contrast, both postmortem studies $^{11,12}$ and electrophysiological studies in aging animals $^{9,19}$ have shown that non-AD processes spare the entorhinal cortex, with selective targeting of other hippocampal regions. Because these regions form a circuit, any lesion can interrupt this circuit and may similarly impair the global function of the hippocampus, as measured by memory tests ${ }^{27}$ or some imaging techniques. Dissociating early $\mathrm{AD}$ from non- $\mathrm{AD}$ causes of memory decline, therefore, may necessitate a regional analysis of the hippocampal formation. Because lesions to hippocampal regions can disrupt the physiological function of hippocampal neurons, and not necessarily result in cell loss, fMRI analysis may detect changes that could be missed by volumetric MRI analysis. ${ }^{28}$ Recent functional imaging techniques, such as positron emission tomography and single-photon emission computed tomography, have detected whole brain activation patterns that discriminate individuals who are at risk for developing $\mathrm{AD}$ dementia, ${ }^{29-31}$ and some studies have found a decrease in global hippocampal function associated with memory impairment. ${ }^{32}$ To date, however, these methods do not possess the spatial resolution to selectively assess different regions within the hippocampal formation and may therefore have difficulty in dissociating processes that result in memory decline by targeting different regions.

\section{Subjects and Methods}

\section{Subjects}

The following three groups of individuals more than 65 years of age participated in the study: (1) 4 subjects with normal memory, (2) 12 subjects with isolated memory decline, and (3) 4 subjects with AD. Subjects from the first two groups were drawn from a single community and were evaluated prospectively with annual medical, neurological, and neuropsychological examinations (the details of this workup are described elsewhere ${ }^{33}$ ). All subjects were presented in a consensus conference composed of neurologists, psychiatrists, and neuropsychologists, and were excluded if at any time point they fulfilled the Diagnostic and Statistical Manual of Mental Disorders ${ }^{34}$ (DSM-IV) criteria for dementia, or if they were diagnosed with stroke, Parkinson's disease, or depression. Subjects were also excluded if they were diagnosed with "questionable dementia," a category applied if neuropsychological test performance was below cutoff scores established for this community, ${ }^{35}$ but were not extensive enough to fulfill dementia criteria. A slope of memory performance over time was calculated for each subject by performing a linear regression of the total recall score of the Selective Reminding Test ${ }^{36}$ a measure of declarative memory. Subjects were assigned to the memory decline group if their memory performance worsened with time; and they were assigned to the normal memory group if their memory did not decline over time. Subjects for the AD group were selected from a clinical setting. They all fulfilled DSM-IV ${ }^{34}$ criteria for dementia, and NINCDS-ADRDA ${ }^{37}$ (National Institute of Neurological and Communicative Disorders and Stroke-Alzheimer's Disease and Related Disorders Association) criteria were used for the diagnosis of probable AD. Only subjects with mild dementia (clinical rating scale ${ }^{38}=1$ ) were selected, based on preliminary studies showing that individuals with more profound dementia could not perform the cognitive activation task.

\section{Task Design}

Stimuli were black-and-white photographs taken from a high school yearbook. All faces were stored digitally in a Macintosh laptop computer and were organized with the Psyscope (Pittsburgh, PA) software program. Faces were projected onto a back-projection screen located at the foot of the magnetic resonance imaging (MRI) bed via an LCD (light- 
emitting diode) projector located outside the scanner room. Subjects viewed the screen via a prism system located in the head coil. All faces were presented one at a time, 4.4 seconds per stimulus, with an interstimulus interval of 0.6 seconds. Responses were recorded with a button box designed for use in the MRI suite.

To accommodate the needs of the subjects, some with memory decline or dementia, the task was kept simple and relatively brief. The task lasted 4 minutes during which an activation phase, made up of 12 faces, alternated with a baseline phase consisting of a fixation point. Task design was as follows: Activation phases occurred during the first and third minutes, and baseline phases during the second and fourth minutes. Thus, in two activation phases a total of 24 different faces were used, with an equal division of sex. Subjects were instructed to push one button if a face was male and the other button if a face was female. All subjects, including the $\mathrm{AD}$ patients, were able to discriminate sex with $100 \%$ accuracy. Left or right button responses that corresponded to sex were pseudorandomized across different subjects within each experimental group. Subjects were instructed to remember the faces for future testing.

\section{Scanning Methods}

Scanning was done on a $1.5-\mathrm{T}$ magnetic resonance scanner retrofitted for echo planar imaging. A gradient echo sequence (echo time $[\mathrm{TE}]=60 \mathrm{msec}$; repetition time $[\mathrm{TR}]=2.5$ seconds; flip angle $=30^{\circ}$ ) and a standard quadrature head coil were used to acquire $\mathrm{T} 2{ }^{*}$-weighted images with an inplane resolution of $2.3 \times 2.3 \mathrm{~mm}\left(64 \times 64\right.$ matrix; $15-\mathrm{cm}^{2}$ field of view). High-resolution "fast multiplanar inversion recovery" images were also acquired, using the same spatial coordinates $(\mathrm{TE}=43 \mathrm{msec} ; \mathrm{TR}=6500 \mathrm{msec}$; inversion time $=200$ msec; $512 \times 512$ matrix; $15-\mathrm{cm}^{2}$ field of view). Six $5-\mathrm{mm}$ slices were selected that were oriented along the long axis of the hippocampal formation. The choice of the most anterior image was based on identification of the alveus and on the image where the temporal horn resides both laterally and superior to the hippocampus proper. ${ }^{39}$ Thus, the most posterior slice was approximately $35 \mathrm{~mm}$ caudal to the amygdala, ensuring incorporation of the entorhinal cortex. ${ }^{40}$

Image analysis was performed on a Silicon Graphics In- digo II workstation, using image display and analysis software packages (MEDx Sensor Systems, Boulder, CO; IDL Research Systems, Sterling, VA). The $\mathrm{AIR}^{41}$ program was applied to correct for head motion and to coregister the volumes. Although the short acquisition time of the runs enhanced the goodness-of-fit of the algorithm head motion is particularly important in this study, as small brain regions were under investigation. Studies were rejected if a shift of $>1 \mathrm{~mm}$ over the scanning time period was detected in any direction after coregistration. Spatial filtering was not used in postacquisition processing, because it effectively reduces both spatial resolution and spatial fidelity.

\section{Data Analysis}

The structural MRI scanning images, which were developed to highlight the internal architecture of the hippocampus (Fig 1), were used to localize three hippocampal regions for each subject - the entorhinal cortex, the hippocampus proper composed of the dentate gyrus and CA subfields, and the subiculum. Fixed anatomical criteria, ${ }^{40-42}$ demonstrated by others to successfully localize hippocampal regions, ${ }^{14,15}$ were used to define these regions by an investigator blinded to experimental grouping. To preserve statistical power, analysis was performed only on pixels within these regions of interest.

Pixel-by-pixel $t$ tests were performed for each subject, comparing the average signal intensity acquired during the activation phases versus the average signal intensity acquired during baseline. Because the goal of this study was to maximally drive the hippocampal formation, the activation and the baseline conditions were purposefully unmatched, since both visual complexity and a motor output contribute to activity in hippocampal neurons. ${ }^{43}$ To account for multiple $t$ testing, we adopted a method developed for multiple singleunit recordings of the hippocampus ${ }^{44}$ to determine an $\alpha$ level that would indicate significant activation. In this method, an $\alpha$ level is chosen, and based on the number of data points sampled, the number of significant data points expected by chance is determined. A $\chi^{2}$ analysis is then performed, comparing the number of observed significant data points with the number expected by chance. In a preliminary series of studies with young and older subjects with normal memory, we found significant hippocampal activation with

Fig 1. Transverse slices through the hippocampal formation. The high-resolution magnetic resonance imaging (MRI) acquisition parameters were specifically developed to highlight the internal architecture of the hippocampal formation. (Right) Example of an MRI slice from 1 subject. (Left) Histological slice of a different hippocampus in approximately the same orientation.
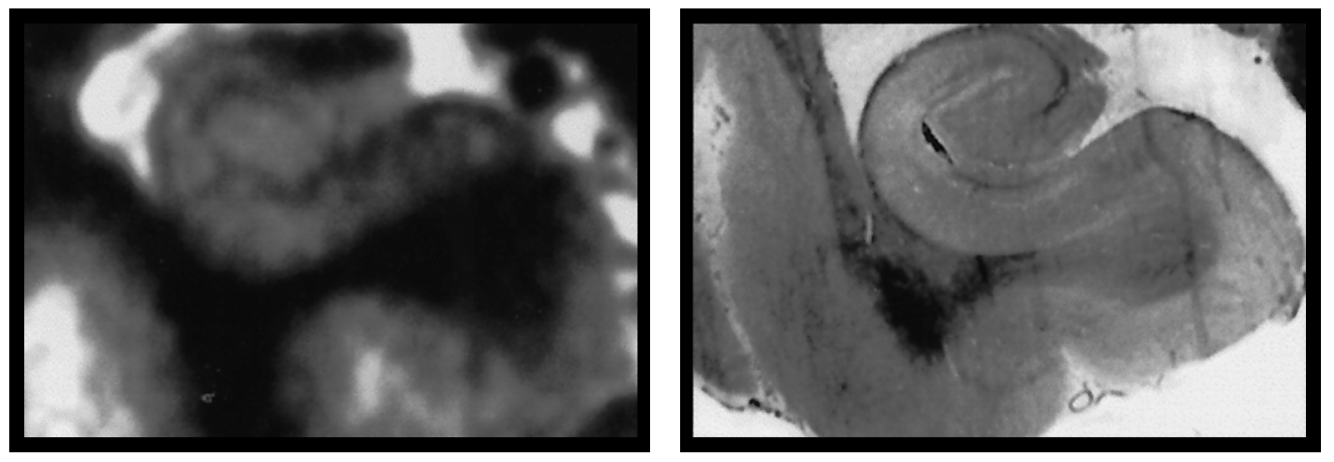
an $\alpha$ of 0.05 . We used this $\alpha$ level for all analyses. To perform group data analysis, an investigator blinded to experimental condition counted the number of pixels whose hemodynamic response significantly increased in association with facial processing. Atrophy was not corrected for because we were interested in activation differences that may result either from structural changes (atrophy and cell loss) or from physiological changes in synaptic efficacy. The number of significant pixels for each hippocampal region was aggregated for each experimental group. In the first experiment, these values were used as the dependent variables in a one-factor multivariate analysis of variance comparing activation between the elderly with normal memory and the patients with probable $\mathrm{AD}$. In the second experiment, subjects with isolated memory decline were dichotomized into two subgroups based on degree of entorhinal activation-subjects with entorhinal activation that was 2 SD less than the normal elderly and those with entorhinal activation that did not differ from the normal elderly. One-factor (group) multivariate analyses of variance were then performed to determine whether these subgroups differed from normal elderly in degree of activation in the hippocampus proper and the subiculum.

\section{Results}

All subjects in the normal elderly group had significant hippocampal activation, determined by $\chi^{2}$ analyses showing a significant $(p<0.0005)$ number of activated pixels compared with the number expected by chance at an $\alpha$ of 0.05 . A limited example is shown in Figure 1. The $\mathrm{AD}$ group was found to be younger (mean, 70.5 years; range, 65-76 years) than the normal memory group (mean, 80 years; range, $73-85$ years). Because increased age is associated with hippocampal atrophy, this may bias the results against finding activation differences. Nevertheless, the $\mathrm{AD}$ subjects were found to have significantly diminished activation compared with normal elderly in all hippocampal regions (entorhinal cortex: $F=22.97, p<0.005$; hippocampal proper: $F=26.72, p<0.001$; subiculum: $F=$ $11.48, p<0.01$ ). A limited example of a subject with $\mathrm{AD}$ is shown in Figure 2. Group data across the three hippocampal regions are shown in Figure 3, which demonstrates no overlap in entorhinal activation between the normal and the $\mathrm{AD}$ groups.

As a group, the mean age of the 12 subjects with isolated memory decline (78.3 years; range, $72-87$ years) was not different from the mean age in subjects with normal memory (80 years; range, $73-85$ years). Results from the second experiment revealed that, of the 12 subjects with memory decline, 4 had diminished entorhinal activation (-EC subgroup) and 8 had normal entorhinal activation (+EC subgroup). Limited examples from each subgroup are shown in Figure 2, and group data are shown in Figure 3. Further analysis revealed that the - EC subgroup had diminished activation in the hippocampus proper $(F=26.72, p<$
$0.005)$ and the subiculum $(F=12.31, p<0.01)$ compared with normal elderly; and that the + EC subgroup had diminished activation restricted to the subiculum $(F=8.99, p<0.01)$.

\section{Discussion}

In this study we present an fMRI protocol developed to perform regional analysis of the hippocampal formation. In the first experiment, we established the regional activation pattern in patients with $\mathrm{AD}$. By the time $\mathrm{AD}$ results in dementia, pathology involves all hippocampal regions. ${ }^{21}$ Finding that $\mathrm{AD}$ subjects had diminished activation in all hippocampal regions compared with normal elderly is, therefore, the expected finding and acts to validate the fMRI protocol used. In the second experiment, we evaluated 12 elderly subjects with a similar behavioral profile, that of declarative memory decline but no dementia. Based on the primacy of entorhinal pathology in $\mathrm{AD},{ }^{10,21,26}$ we predicted that these subjects could be dichotomized on the basis of entorhinal function. Indeed, we found that 4 of these subjects had entorhinal dysfunction (the -EC subgroup) whereas 8 of them had normal entorhinal function (the +EC subgroup). Among the $-\mathrm{EC}$ subgroup, further analysis revealed diminished activation in all hippocampal regions, and their regional activation pattern was indistinguishable from the pattern generated in patients with $\mathrm{AD}$. Because the entorhinal cortex is the first subregion of the hippocampal circuit, a lesion here would be expected to result in diminished activity throughout the circuit. Alternatively, it is possible that the the presumptive AD pathology in these subjects had already progressed beyond the entorhinal cortex to include other hippocampal regions. We are currently evaluating extrahippocampal sites in the temporal and parietal cortices and predict that only $A D$ patients will manifest diminished activation in these neocortical sites. We are also evaluating the apolipoprotein $\mathrm{E}$ genotype of all subjects with memory decline, to determine whether the $-\mathrm{EC}$ subgroup has an increased frequency of the apolipoprotein E 4 allele.

The subgroup of subjects with hippocampal-based memory decline and preserved entorhinal function are unlikely to have early AD. Nevertheless, these subjects were found to have hippocampal dysfunction, but diminished activation was restricted to the subiculum. Selective subicular dysfunction may result from physiological or structural lesion to this region, which would fit well with recent postmortem findings from brains without $\mathrm{AD}$, showing age-dependent subicular cell loss. ${ }^{11,12}$ Alternatively, diminished activation in the subiculum may arise from lesions upstream in the hippocampal circuit in the CA regions, where animal studies have demonstrated age-related physiological deficits. ${ }^{9,19}$

Prospective follow-up will validate whether those 


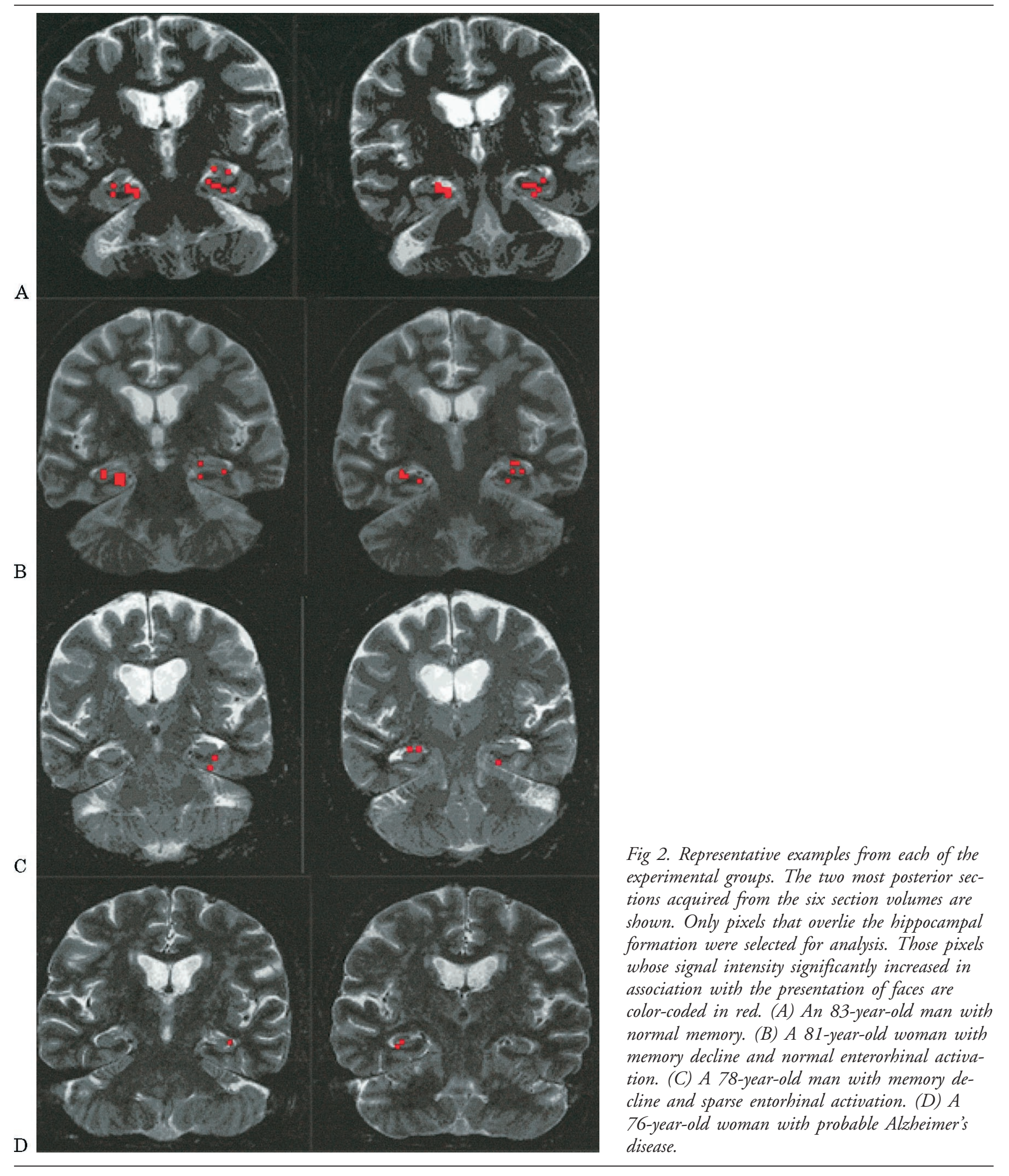

subjects with memory decline and entorhinal dysfunction are more likely to progress to $\mathrm{AD}$ dementia compared with those subjects with normal entorhinal function. If so, the fMRI protocol presented in this study will benefit both $\mathrm{AD}$ research and the study of non-AD memory decline. The rationale behind current interventions of $\mathrm{AD}$ is halting disease progression, and therefore, a major goal is early detection. The fMRI protocol may be developed as a tool for detecting $\mathrm{AD}$ at its earliest stages, where it presents with mild memory decline. The rigorous study of non-AD age-related memory decline has been hindered, and remains a con- 


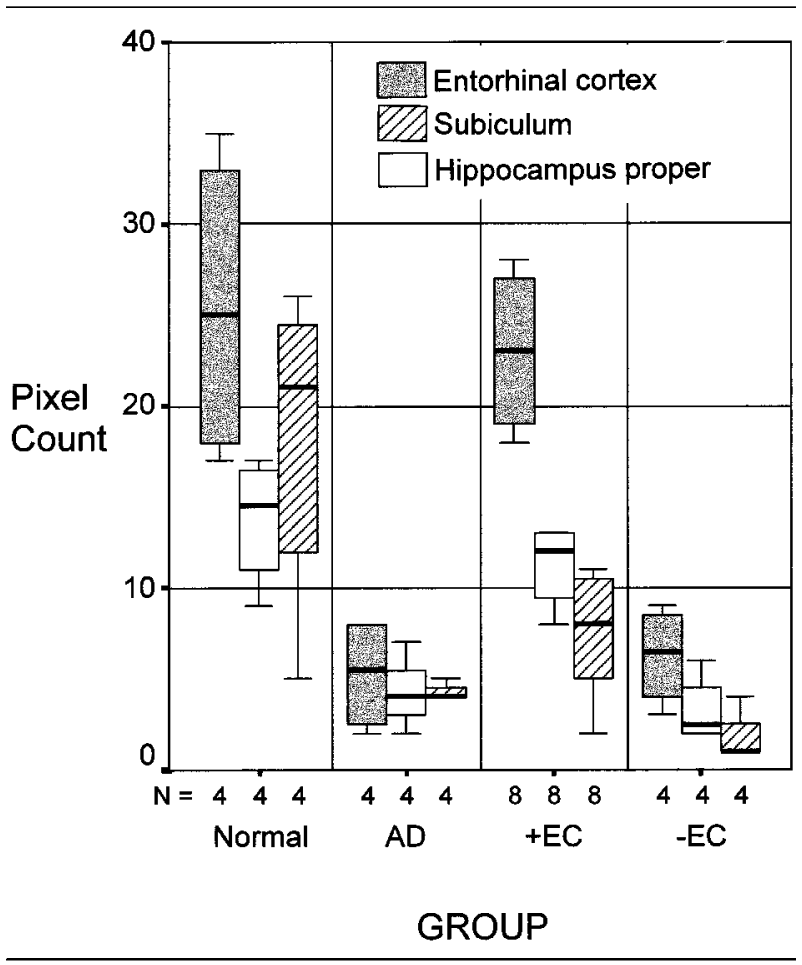

Fig 3. A box plot of group data comparing the number of significantly activated pixels in each hippocampal region across the four groups-normal elderly, Alzheimer's disease (AD), isolated memory decline with normal entorhinal function $(+E C)$, and isolated memory decline with entorhinal dysfunction $(-E C)$. (Single outliers in the entorhinal cortex [EC] and hippocampus proper of the $+E C$ group are not shown.)

troversial entity, in large part because of the inability to select out those subjects with early AD. The fMRI protocol may also be used to isolate a group of elderly individuals with non- $\mathrm{AD}$ memory decline. This will allow better characterization of its clinical presentation and course, and the testing of candidate processes as underlying etiologies.

In summary, the results of this study show that agerelated memory decline is associated with hippocampal dysfunction. More important, regional analysis of the hippocampal formation provided direct evidence that age-related memory decline is not caused by a singular process. Showing that AD subjects and a subset of subjects with memory decline have an indistinguishable pattern of regional dysfunction supports the likelihood that $\mathrm{AD}$ is one cause of memory decline. A second pattern of regional dysfunction, however, found in a different subset of subjects with memory decline, is difficult to reconcile with the established distribution of $\mathrm{AD}$ pathology. Non-AD processes that selectively target the hippocampal proper and the subiculum can more easily account for this regional pattern. Both early $\mathrm{AD}$ and non- $\mathrm{AD}$ processes, therefore, are implicated as underlying causes of age-related memory decline.
This research was supported by federal grants AG08702, AG07232, and AG01963

\section{References}

1. Scoville WB, Milner B. Loss of recent memory after bilateral hippocampus lesions. J Neurol Neurosurg Psychiatry 1957;20: $11-21$

2. Rempel-Clower NL, Zola-Morgan S, Squire LR, Amaral DG. Three cases of enduring memory impairment following bilateral damage limited to the hippocampal formation. J Neurosci 1996; 16:5233-5255

3. Jarrard LE. On the role of the hippocampus in learning and memory in the rat. Behavioral and recognition in the human brain. Proc Natl Acad Sci USA 1996;93:922-927

4. Squire LR, Ojemann JG, Miezin FM, et al. Activation of the hippocampus in normal humans: A functional anatomical study of memory. Proc Natl Acad Sci USA 1992;89:1837-1841

5. Stern CE, Corkin S, Gonzalez RG, et al. The hippocampal formation participates in novel picture encoding: evidence from functional magnetic resonance imaging. Proc Natl Acad Sci USA 1996;93:8660-8665

6. Amaral DG, Witter MP. The three dimensional organization of the hippocampal formation: a review of anatomical data. Neuroscience 1989;31:571-591

7. Bliss TVP, Lomo T. Long-lasting potentiation of synaptic transmission in the dentate area of the anaesthetized rabbit following stimulation of the perforant pathway. J Physiol (Lond) 1973;232:331-356

8. Nicoll RA, Malenka RC. Contrasting properties of two forms of long term potentiation in the hippocampus. Nature 1995; 377:115-118

9. Barnes CA. Normal aging: regionally specific changes in hippocampal synaptic transmission. Trends Neurosci 1994;17: $13-18$

10. Gomez-Isla T, Price JL, McKeel DW, et al. Profound loss of layer II entorhinal cortex neurons occurs in very mild Alzheimer's disease. J Neurosci 1996;16:4491-4500

11. West MJ. Regionally specific loss of neurons in the aging human hippocampus. Neurobiol Aging 1993;14:287-293

12. Simic G, Kostovic I, Winblad B, Bogdanovic N. Volume and number of neurons of the human hippocampal formation in normal aging and Alzheimer's disease. J Comp Neurol 1997; 379:482-494

13. Kwong KK, Belliveau JW, Chesler DA. Dynamic magnetic resonance imaging of human brain activity during primary sensory stimulation. Proc Natl Acad Sci USA 1991;88:5829-5831

14. Mori E, Yondea Y, Yamashita H, et al. Medial temporal lobe structures related to memory impairment in Alzheimer's disease: an MRI volumetric study. J Neurol Neurosurg Psychiatry 1997; 63:214-221

15. Gabrieli JDE, Brewer JB, Desmond JE, Glover GH. Separate neural bases of two fundamental memory processes in the human medial temporal lobe. Science 1997;276:264-266

16. Koivisto K, Reiniainen KJ, Hanninen T, et al. Prevalence of age-associated memory impairment in a randomly selected population from eastern Finland. Neurology 1995;45:741-747

17. Petersen RC, Smith G, Kokmen E, et al. Memory function in normal aging. Neurology 1992;42:396-401

18. Craik FIM, Anderson ND, Kerr SA, Li JZH. Memory changes in normal ageing. In: Baddely AD, Wilson BA, Watts FN, eds. Handbook of memory disorder. West Sussex, UK, Wiley and Sons, 1995:211-241

19. Geinisman Y, Detoledo-Morrell L, Morrell F, Heller RE. Hippocampal markers of age-related memory dysfunction: behavioral electrophysiological and morphological perspectives. Prog Neurobiol 1995;45:223-252 
20. Rapp PR, Amaral DG. Individual differences in the cognitive and neurobiological consequences of normal aging. Trends Neurosci 1992;15:340-345

21. Braak H, Braak E. Evolution of the neuropathology of Alzheimer's disease. Acta Neurol Scand Suppl 1996;165(Supp):3-12

22. Jacobs DM, Sano M, Dooneief G, et al. Neuropsychological detection and characterization of preclinical Alzheimer's disease. Neurology 1995;45:957-962

23. Belanger A, Candas B, Dupont A, et al. Changes in serum concentrations of conjugated and unconjugated steroids in 40 to 80 year old men. J Clin Endocrinol Metab 1994;79:10861090

24. de la Torre JC, Park G, Fortin T, et al. Loss of hippocampal neurons after chronic brain hypoperfusion in aged rats. Neurobiol Aging 1990;11:328-329

25. Lupien SJ, de Leon M, de Santi S, et al. Cortisol levels during human aging predict hippocampal atrophy and memory deficits. Nat Neurosci 1998;1:69-73

26. Masiliah E, Mallory M, Hansen L, et al. Synaptic and neuritic alterations during the progression of Alzheimer's disease. Neurosci Lett 1994;174:67-72

27. Morris RGM, Schenk R, Tweedle F, Jarrard LE. Ibotenate lesions of hippocampus and/or subiculum: dissociated components of allocentric spatial learning. Eur J Neurosci 1990;2: $1016-1028$

28. Golomb J, Kluger A, de Leon MJ, et al. Hippocampal formation size in normal human aging: a correlate of delayed secondary memory performance. Learn Memory 1994;1:45-54

29. Small GW, Mazziotta JC, Collins MT, et al. Apolipoprotein E type 4 allele and cerebral glucose metabolism in relatives at risk for familial Alzheimer disease. JAMA 1995;273:942-947

30. Reiman EM, Caselli RJ, Yun LS, et al. Preclinical evidence of Alzheimer's disease in persons homozygous for the epsilon 4 allele for apolipoprotein E. N Engl J Med 1996;334:752-758

31. Johnson KA, Jones K, Holman BL, et al. Preclinical prediction of Alzheimer's disease using SPECT. Neurology 1998;50:15631571

32. Grady CL, McIntosh AR, Horwitz B, et al. Age-related reduc- tions in human recognition memory due to impaired encoding. Science 1995;269:218-221

33. Stern Y, Gurland B, Tatemichi TK, et al. Influence of education and occupation on the incidence of Alzheimer's disease. JAMA 1994;271:1004-1010

34. American Psychiatric Association. Diagnostic and statistical manual of mental disorders 4. Washington, DC: American Psychiatric Association, 1994

35. Stern Y, Andrews H, Pittman J, et al. Diagnosis of dementia in a heterogenous population. Arch Neurol 1992;49:453-460

36. Buschke H, Fuld PA. Evaluating storage, retention, and retrieval in disordered memory and learning. Neurology 1974;24: 1019-1025

37. McKhann G, Drachman D, Folstein M, et al. Clinical diagnosis of Alzheimer's disease: report of the NINCDS-ADRDA work group under the auspices of Department of Health and Human Services task force on Alzheimer's disease. Neurology 1984;34:939-944

38. Hughes C, Berg L, Danziger W, et al. A new clinical scale for the staging of dementia. Br J Psychiatry 1982;140:566-572

39. Woods RP, Mazziotta JC, Cherry SR. MRI-PET registration with automated algorithm. J Comput Assist Tomogr 1993;17: 536-546

40. Duvernoy HM. The human hippocampus: an atlas of applied anatomy. Munich, Germany: JF Bergman, 1988

41. Amaral DG, Insausti R. The hippocampal formation. In: Paxinos G, ed. The human nervous system. San Diego: Academic Press, 1990:711-755

42. Francis H, Cavazos JE, Tien RD. Hippocampus: normal magnetic resonance imaging anatomy with volumetric studies. Neuroimaging Clin N Am 1997;7:11-30

43. Halgren E, Babb TL, Crandall PH. Activity of human hippocampal formation and amygdala neurons during memory testing. Electroencephalogr Clin Neurophysiol 1978;45:585601

44. Fried I, MacDonald KA, Wilson CL. Single neuron activity in human hippocampus and amygdala during recognition of faces and objects. Neuron 1997;18:753-765 